\title{
A Light Dark Matter signature?
}

\author{
Céline Bœhm* \\ Astrophysics Department, University of Oxford \\ 1 Keble Road, OX1 $3 R H$ Oxford, UK \\ E-mail: 'boehmQastro.ox.ac.uki
}

ABstract: We discuss the possibility of having Light Dark Matter particles and present a possible signature in favor of such particles. We also explain how they could evade the Lee-Weinberg limit, past accelerator searches as well as astrophysics constraints.

\section{Introduction}

The lightest neutralino $\chi^{0}$ is known to be one of the most appealing Dark Matter (DM) candidate. This linear combination of the supersymmetric partners of neutral Higgs and gauge bosons is stable if it is the Lightest Supersymmetric Particle (LSP) and if $R$-parity is conserved [i]. However, there is no evidence in favor of supersymmetry so far, so there is still room for alternative DM candidates. This is the question we shall address in this paper; in particular we shall consider whether there is any other particle that could solve the DM problem.

To be a viable solution, a DM candidate must account for $\sim 25 \%$ of the content of the

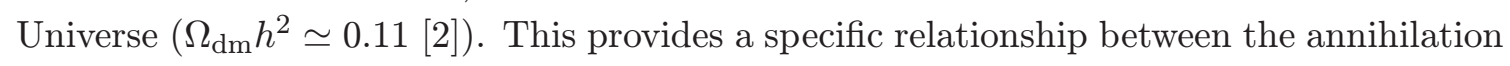
cross section and other DM parameters:

$$
\sigma v \simeq 7 \times 10^{-27} \times \frac{x_{F}}{\sqrt{g_{\star}}}\left(\frac{\Omega_{\mathrm{dm}} h^{2}}{0.11}\right)^{-1} \mathrm{~cm}^{3} \mathrm{~s}^{-1},
$$

where $x_{F} \sim 12-22$ for particles in the $\mathrm{MeV}-100 \mathrm{GeV}$ range respectively and $g_{\star}$ the number of relativistic degrees of freedom. If the total annihilation cross section is much lower, then there is too much DM; if it is much larger, then there is not enough left (see for example [3. DM alternatives.

This formula is almost independent of the DM mass. In fact, the only significant dependence in $m_{d m}$ is potentially through the DM annihilation cross section, as we shall discuss later. This indicates that one can both consider particles lighter than a few $\mathrm{GeV}$

\footnotetext{
${ }^{*}$ Speaker.
} 
and satisfy the relic density criterion. However, light DM particles are in danger to yield an overproduction of neutrinos or photons at an epoch where nucleosynthesis takes (or has already taken) place. We shall therefore restrict our attempt to find DM alternatives in the mass range above a few $\mathrm{MeV}$ (say $m_{d m} \in[\mathrm{MeV}, \mathrm{GeV}]$ ).

Although heavy enough, Light DM particles could nevertheless affect structure forma-

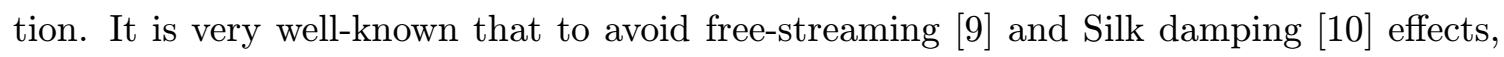
DM should have a mass larger than a few $\mathrm{keV}$ (as confirmed from numerical simulations

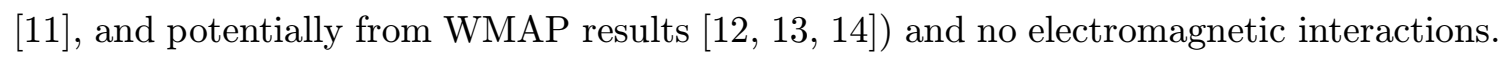
Altogether, these two conditions suggest that DM should be massive and weakly interacting, so any alternative should get these two properties or at least not suffer from too large damping effects.

In section I, we shall review the conditions for the existence of Light DM particles from structure formation. We then investigate whether light DM indeed corresponds to Weakly Interacting Massive Particles (WIMPs) and discuss their possible signatures on linear and non-linear matter power spectra. In the second section, we give details on how to evade the Lee-weinberg limit. We then check out the constraints from direct and indirect detection experiments and finally define the particle physics properties that a light DM candidate should get to be viable solution. We end up by giving an interesting feature for Light DM particles.

\section{Does structure formation allow for surprising candidates?}

It is often said that the precise measurement of the Cosmic Microwave Background (CMB) up to the second peak favors the CDM scenario, defined as being made of particles with no or negligible interactions. However, the maximum value of the DM cross section compatible with both $\mathrm{CMB}$ and structure formation turns out to be much larger than what is naively

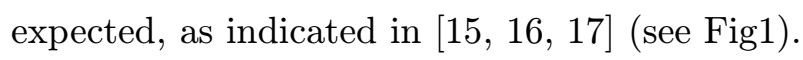

For example, by simulating the effect of $\mathrm{dm}-\gamma$ interactions on the CMB, one can show that even unrealistic value of this interaction (say up to $\sigma_{d m-\gamma} \sim 10^{-30} \mathrm{~cm}^{2}$ for $m_{d m} \sim$ $\mathrm{GeV}$ ) does not modify the CMB spectrum on visible scales (at least not on the scales that have been measured by WMAP, Boomerang or other CMB experiments). Similarly, even such a very large value of the DM- $\gamma$ cross section affects the linear matter power spectrum on scales smaller than $10^{9} M_{\odot}$ (which is the present state of observations, excluding however lensing measurements of halo substructures). As a result, even very large DM interactions seem perfectly compatible with observations, albeit quite unrealistic from a particle physics point of view. This, in principle, puts in perspective the notion of WIMP, since even quite "strongly" DM particles seem to be a possible alternative to supersymmetric DM from structure formation.

It is quite interesting to note that DM interactions (in particular with photons) yield a damping of the linear matter power spectrum that manifests itself under oscillations. This may be used as a signature to discriminate among collisionless Warm Dark Matter scenarios (WDM) and more sophisticated scenarios where the DM fluctuations are damped below a scale determined by the ratio of the DM cross section to its mass. 

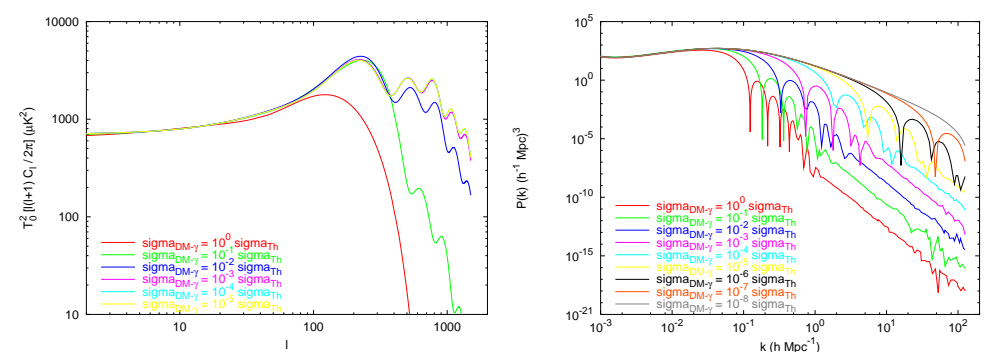

Figure 1: power and matter power spectra for interacting DM with photons [1]

In fact, in the case of DM- $\gamma$ interactions, DM simply has to decouple from photons before the epoch of matter-radiation equality to be compatible with data. This condition may be improved from the measurement of structures and substructures at very smallscales nevertheless. But so far, one is still allowed to introduce a set of new and surprising interacting candidates.

As already mentioned, galaxy surveys and/or lensing measurements may actually be able to discriminate among Cold and WDM-like scenarios through the measurement of the non-linear matter power spectrum. It is therefore important to determine the non-linear matter power spectra of WDM-like scenarios and compare them to CDM spectra. Some of our results are presented in Figure?프.
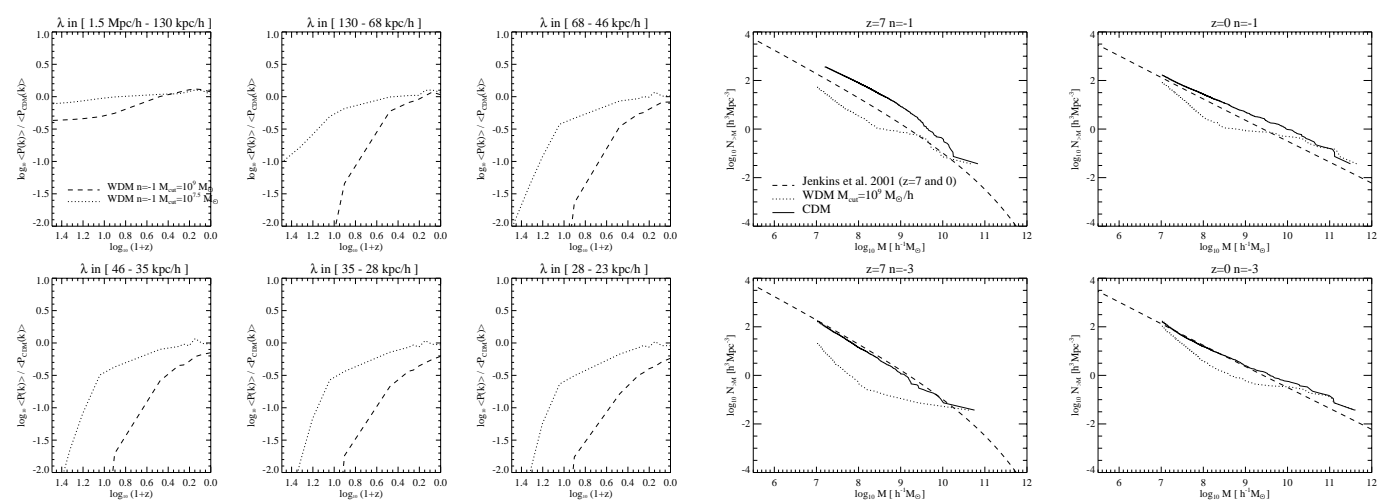

Figure 2: On the top: ratio of CDM and WDM matter power spectra for a box of $1.5 \mathrm{Mpc} / \mathrm{h}$ depending on the redshift. On the bottom: halo mass functions for a box of $1.5 \mathrm{Mpc} / \mathrm{h}$ depending on the redshift [1] $\overline{1} \overline{1}]$.

One readily sees that, due to a small-scale generation mechanism, even the non-linear matter power spectrum of $1 \mathrm{keV}$ WDM particle seems compatible with a Cold Dark Matter scenario. It is therefore unlikely that one may be able to distinguish between a Warm and Cold DM scenario from the observation of the non-linear matter power spectrum only [1] (see left panel Figi2i). Finally, other signatures, like the number of halo substructures for example, may be used to determine the nature of DM. However only semi-analytic estimates have been given so far. Their predictions might differ from numerical simulations so we let this point as an open question which would deserve a quite detailled study. 
Let us precise also that DM- $\nu$ interactions can also yield a significant damping. However, the ratio of the DM cross section to its mass that yields a damping below $100 \mathrm{kpc}$ (corresponding to $10^{9} M_{\odot}$ ) is of

$$
\sigma_{d m-\nu}=10^{-35}\left(\frac{m_{d m}}{\mathrm{GeV}}\right)\left(\frac{l}{100 \mathrm{kpc}}\right)^{2} \mathrm{~cm}^{2}
$$

For $m_{d m} \lesssim \mathrm{GeV}$, the values of $\sigma_{d m-\nu}$ that can generate the collisional damping are of weak intensity. Therefore, it seems like even WIMP particles can suffer from large damping effects! The latter may not be dramatic because of the small-scale generation mechanism but certainly deserve to be taken into account. One should therefore be careful when doing numerical simulations as the latter may be incorrect if one neglects the DM interactions without a preliminary check!

To conclude this section, alternative DM scenarios may therefore be allowed, even if they yield a damping at a significant scale (say $10^{6}-10^{9} M_{\odot}$ ). In the scenario we shall consider on the other hand this damping is at most $10^{3} M_{\odot}$, so they should be safe from present observational constraints.

Finally, we have seen that i) structure formation could allow for alternative candidates, provided some conditions on the DM mass and cross sections, and ii) DM particles of a few $\mathrm{GeV}$ (or even lighter) could even yield challenging questions for numerical simulations depending on the DM interactions. BUT can Dark Matter be as light (or even lighter) than a few $\mathrm{GeV}$ ?

\section{Relic density: how to evade the Lee-Weinberg limit?}

The precise measurement of DM cosmological parameters [i] 1$] 1$ indicates that DM represents $\sim 25 \%$ of the content of the Universe, corresponding to the DM cosmological parameter $\Omega_{d m} h^{2}=\frac{n_{d m} m_{d m}}{\left(\rho_{c} / h^{2}\right)} \sim 0.11$. This turns out to be a stringent condition for any DM candidates, including neutralinos, as shown below.

The number density $\left(n_{d m}\right)$ of DM particles satisfies the following Boltzmann equation:

$$
\frac{d n}{d t}=-3 H n_{d m}-\langle\sigma v\rangle\left(n_{d m}^{2}-n_{e}^{2}\right)
$$

where $\langle\sigma v\rangle$ is the annihilation cross section (here we disregard possible coannihilation processes). At very high energy, say $T>m_{d m}$, the number of DM particles (which are supposed to be in thermal equilibrium) is proportional to $T^{3}$ (with $T$ the temperature of the thermal bath). Annihilations start after DM became non-relativistic. More precisely, they start when the temperature of the Universe drops below $T \sim m_{d m} / 3$ (provided that the relationship $\langle\sigma v\rangle n_{d m}>H$ is satisfied). The comoving DM number density (in which one has removed the expansion effects) exponentially decreases. This is what prevents the DM to overclose the Universe. When $H$ becomes $\sim\langle\sigma v\rangle n_{d m}$, the annihilations reactions freezeout and the DM comoving number density is conserved till nowadays, so $n\left(T_{f o}\right) a^{3}\left(T_{f o}\right)=$ $n\left(T_{0}\right)$. By using the following relationship,

$$
\begin{aligned}
& \Omega_{d m}=\frac{\rho_{d m}}{\rho_{c}}=n_{d m}\left(T_{0}\right) m_{d m} \\
& \text { and } \quad n_{d m}\left(T_{0}\right)=n_{d m}\left(T_{f o}\right) a_{f o}^{3}, \quad H_{f o} \propto a_{f o}^{-2}, \quad a_{f o} \sim T_{0} / T_{f o}
\end{aligned}
$$


one finally gets

$$
(\sigma v)_{a n n} \sim 710^{-27} \frac{x_{f o}}{\sqrt{g}_{\star}}\left(\frac{\Omega_{d m} h^{2}}{0.1}\right)^{-1} \mathrm{~cm}^{3} \mathrm{~s}^{-1} .
$$

The DM relic density is therefore expected to be compatible with WMAP results if the DM annihilation cross section is about $\sigma v \simeq 710^{-27} \frac{x_{f o}}{\sqrt{g_{x}}} \mathrm{~cm}^{3} \mathrm{~s}^{-1}$. Note that this relationship has been obtained independently of any DM candidate. In particular, it is independent of $m_{d m}$ ! This means that any kind of DM candidate (light or not) can have the correct relic density if its annihilation cross section has the correct value. There are more sophisticated ways to solve the Boltzmann equation, in particular, by using numerical calculations. However, we do not need such accurate treatment to discuss the possibility of Light DM so we shall use the approximate solution given above.

As a first illustration, one can discuss the annihilation cross section of neutralinos DM. Despite many exceptions (for example resonance situations), one can write their annihilation cross section as

$$
\sigma v_{a n n} \propto 10^{-27}\left(\frac{c}{\alpha}\right)^{2}\left(\frac{m_{d m}}{100 \mathrm{GeV}}\right)^{2}\left(\frac{m_{F}}{100 \mathrm{GeV}}\right)^{-4} \mathrm{~cm}^{3} / \mathrm{s},
$$

where $c$ is the couplings and $m_{F}$ the mass of the particles exchanged during the annihilation process $\left(m_{F}>100 \mathrm{GeV}\right.$ if $F$ are charged particles, as indicated from unfruitful accelerator searches). Neutralinos are therefore likely to get the correct relic density if their mass is about $100 \mathrm{GeV}$. In fact, one gets an upper limit on the neutralino mass when $m_{F} \sim m_{d m}$ (since in this case the cross section is inversely proportional to $m_{d m}$ ) and a lower limit on $m_{d m}$, depending on $m_{F}$ and the couplings, when $m_{F}$ is as close as it can be to its experimental exclusion limit.

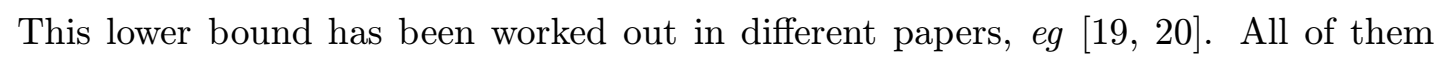
confirm the Lee-Weinberg limit, following which $m_{d m}$ must be greater than a few $\mathrm{GeV}$ to satisfy the relic density condition. As we have seen, nevertheless, the relic density criterion allows for any kind of DM particles as long as their annihilation cross section is about $10^{-26} \mathrm{~cm}^{3} \mathrm{~s}^{-1}$. Therefore, one should be able to evade the Lee-Weinberg limit, just by considering a cross section that is independent of $m_{d m}$. But is there any particle for which the annihilation cross section does not depend on the DM mass? One may try a simple attempt, i.e considering scalar DM particles (by opposition to fermions) that would exchange a heavy fermion $F$.

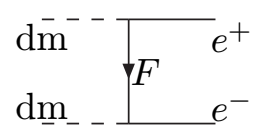

One obtains the following annihilation cross section:

$$
\sigma v \propto\left(1-\frac{m_{f}^{2}}{m_{d m}^{2}}+v_{d m}^{2}\right) \frac{c_{l}^{2} c_{r}^{2}}{m_{F}^{2}} \sim \frac{c_{l}^{2} c_{r}^{2}}{m_{F}^{2}}
$$


which is remarkably independent of $m_{d m}$ ! This therefore confirms that it is possible to evade the Lee-Weinberg limit and that one can consider particles lighter than a few GeV, at least as long as they are scalars! One then has to check whether such particles could have been observed in direct, indirect detection experiments or in accelerator searches. In which case, they would finally be excluded.

\section{Direct Detection}

Most of direct detection experiments are based on nucleus recoil, ionization and/or scintillation. They turn out to be quite sensitive to large masses and therefore appear quite appropriate to detect heavy WIMPs. No positive signal has been detected so far so this constrains the supersymmetric phase space. There is nevertheless one experimental claim in favor of heavy DM particles through annual modulation [i2 in] . However, this result is quite controversial, in particular because it is in contradiction with Edelweiss experiment. These two experiments use different techniques and detectors nevertheless so it is difficult to compare them directly.

All direct experiments are supposed to be unsensitive to particles lighter than a few $\mathrm{GeV}$ since the collision of very light particles with the nuclei present in the detector would release too few energy to be detectable. Some experiments, like CRESST, MACHE3, ROSEBUD, PICASSO, etc, do have a very low energy threshold nevertheless. Although this may help to detect DM particles lighter than $10 \mathrm{GeV}$, particles much lighter than a few $\mathrm{GeV}$ should still be hard to detect anyway, unless perhaps one focuses on their interactions with electrons. However, this has not been studied so far.

\section{Indirect detection}

Another important experimental constraint concerned indirect detection. Similarly to neutralinos, Light DM particles can annihilate into $e^{+} e^{-}, \gamma \gamma$ etc. This leads to a specific signature at low energy that may be used to constrain the DM properties. This contrasts with neutralinos which could perhaps be at the origin of the positron excess (as detected by HEAT experiment between $[8,20] \mathrm{GeV}$ ) and very high energy gamma ray lines ${ }^{1}$ albeit this is under debate, as discussed in [23] , and somewhat in contradiction with the extrapolation of the Milky Way dark halo profile from lensing observations (which favor a $\sim$ flat profile

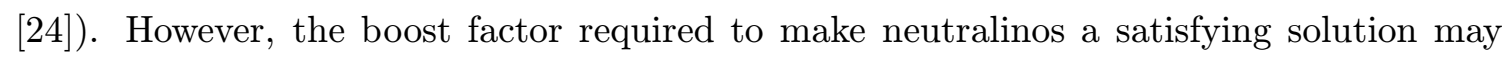
still be explained by invoking a black hole in the inner region of our galaxy.

Let us come back to Light DM. Since the DM number density is inversely proportional to the DM mass, one expects the gamma ray flux from the center of the Milky Way to be larger in the case of Light DM than in the case of neutralinos (as the flux is proportional to the square of the DM number density). This actually turns out to constrain Light DM mass and annihilation cross section as gamma rays in the $[\mathrm{MeV}, \mathrm{GeV}]$ energy range have already been well measured [i2 $2 \overline{5} \overline{1}]$.

\footnotetext{
${ }^{1}$ See for example, the recent claim [22] explain the observed excesses, provided that the Milky Way Dark halo profile is a Moore profile.
} 
For example, at recombination, one obtains a flux which is close to observational limits but still not forbidden. The constraints one obtains from the center of our galaxy are much more strigent on the other hand. To estimate the flux, one can parameterize the Milky Way DM halo profile as

$$
\rho(r)=\frac{\rho_{0}}{\left(\frac{r}{r_{s}}\right)^{\gamma}\left(1+\left(\frac{r}{r_{s}}\right)^{\alpha}\right)^{-\frac{(\beta-\gamma)}{\alpha}}} .
$$

We shall consider two extreme cases, namely Navarro-Frenk-White (which corresponds to a cuspy halo) and Binney-Evans (which corresponds to a rather flat profile, i.e with a core) profiles. The gamma ray flux is then given by:

\begin{tabular}{|l|l|l|l|l|l|l|}
\hline & $\alpha$ & $\beta$ & $\gamma$ & $r_{s}(\mathrm{kpc})$ & $\phi_{t h}\left(<1.5^{\circ}\right)$ \\
\hline$N F W$ & 1 & 3 & 1 & 25 & $610^{-6}\left(\frac{m_{d m}}{\mathrm{GeV}}\right)^{-2}\langle\sigma v\rangle_{26}$ \\
\hline Binney - Evans & 1 & 3 & 0.3 & 4 & $410^{-6}\left(\frac{m_{d m}}{\mathrm{GeV}}\right)^{-2}\langle\sigma v\rangle_{26}$. \\
\hline
\end{tabular}

To compare with, the observed flux is about $\phi_{t h}\left(<1.5^{\circ}, E \gtrsim \mathrm{MeV}\right)=10^{-5} \mathrm{~cm}^{2} \mathrm{~s}^{-1}$. The latter being fairly understood, $\mathrm{MeV}$ particles are likely to be excluded if their annihilation cross section inside the DM halo is about $10^{-26} \mathrm{~cm}^{3} \mathrm{~s}^{-1}$ ! However, in the above table, we supposed that the annihilation cross section behaved as a constant. Said differently, using the following decomposition $\sigma v \propto a+b v^{2}$, our previous calculations supposed $a>>b$. However, if the annihilation cross section turns out to behave instead as $\sigma v \propto b v^{2}$ (where we now assume $a<<b v^{2}$, bearing in mind that the DM velocity in the galaxy is $10^{-3} c$ ), then $\mathrm{MeV}$ particles appears acceptable DM candidates. These constraints are less stringent for $100 \mathrm{MeV}$. Heavier particles seem indeed marginally acceptable, without the need for $v^{2}$ annihilation cross section.

We are now left with a very important question: Can $\mathrm{MeV}$ particles have a $v^{2}$ suppressed annihilation cross section? This was studied in great details in [2] $\left.{ }_{2}^{2} \overline{6}\right]$. We have seen previously that one could evade the Lee-Weinberg limit by invoking scalar DM particles. If the annihilation mechanism proceeds through a fermion exchange nevertheless, then the annihilation cross section behaves as

$$
\sigma v \propto\left(1-\frac{m_{f}^{2}}{m_{d m}^{2}}+v_{d m}^{2}\right) \frac{c_{l}^{2} c_{r}^{2}}{m_{F}^{2}} .
$$

This expression indicates that both $a$-term and $b$-term behave in the same way so it is not possible to obtain $a \ll b$, as needed to satisfy the gamma ray constraint. Another solution consists in introducing a new gauge boson. The annihilation process can then proceeds through the boson exchange rather than a fermion exchange.

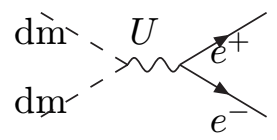


The associated cross section is given by

$$
\sigma v \propto v_{d m}^{2} C_{U}^{2} f_{U}^{2}\left(\frac{m_{d m}^{2}}{m_{U}^{4}}\right)
$$

which appears $v^{2}$-suppressed. By introducing an extra gauge boson, we then managed to obtain a $v^{2}$-supressed annihilation cross section, as required by the gamma ray constaint but, on the other hand, we also introduced the $m_{d m}$ dependence that we wanted to avoid!

This dependence is no longer a problem, however, as the gauge boson can be much lighter than $100 \mathrm{GeV}$ ! Indeed the experimental limits on the existence of such particles are less restricted, especially due to the fact they are neutral and should be weakly coupled (as we shall discuss in the next section).

We therefore succeed to build a model in which the DM is i) light, ii) in agreement with gamma ray constraints and the relic density condition. The existence of MeV DM seems possible provided the major caveat that it is made of scalar particles and coupled to a light gauge boson very weakly coupled to Standard Model particles! In fact, there may be other viable solutions but we do not discuss them in the present paper.

\section{Particle Physics constraints}

We saw in previous sections that it is possible to build a model for Light DM that satisfies several astrophysical constraints. In the above illustration, we introduce a scalar particle and a new light gauge boson. We now have to show that this solution is not experimentally excluded. We also need to explain why such particles could not be detected in previous accelerator searches.

Unlike neutralinos which are hard to detect or to exclude given the impressive number of free parameters in supersymmetric theories, Light DM particles appear extremely constrained. There are many experiments which look for neutral and stable particles, in particular through Initial State Radiation processes $\left(e^{+} e^{-} \rightarrow \gamma+\right.$ missing energy), as used in neutrino and neutralino searches. No anomalous single photon events have been found so this may be used as a constraint. Also, a light gauge boson $U$ or DM particles could give an extra contribution to the muon and electron $g-2$, or to the $\nu-e$ elastic scattering cross section, so this restricts the parameter space available for Light DM.

The extra contributions of a new light gauge boson to the muon and electron $g-2$ as well as to the $\nu-e$ elastic scattering cross section turn out to be the most stringent constraints.

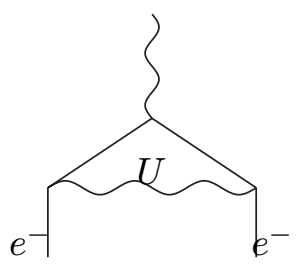


By comparing the extra contribution from an extra gauge boson to the muon and electron $g-2$, namely

$$
\delta a_{\mu>m_{U}}=\frac{f_{U l}^{2}}{8 \pi^{2}}
$$

(where we assume vectorial couplings, say $f_{U l}=f_{U r}$ ) and

$$
\delta a_{m_{U}>m_{e}}=\frac{f_{U l}^{2}}{12 \pi^{2}} \frac{m_{e}^{2}}{m_{U}^{2}},
$$

to experimental limits, say: $\delta a_{\mu} \lesssim 10^{-9}$ and $\delta a_{e} \lesssim 10^{-11}$, one obtains a constraint on the coupling between the gauge boson and electrons (and also muons, but we do not discuss it here), namely:

$$
f_{U e}=710^{-5}\left(\frac{m_{U}}{\mathrm{MeV}}\right)\left(\frac{\delta a_{e}}{10^{-11}}\right)^{1 / 2},
$$

which, depending on $\delta a_{e}$, could be extremely small. Using now the relic density criterion (and more precisely the value of the annihilation cross section), one obtains a precise value of the product of the coupling of the gauge boson with DM and electrons, say:

$$
C_{U} f_{U e}=(3-12) 10^{-8}\left(\frac{m_{U}}{\mathrm{MeV}}\right)^{2}\left(\frac{m_{d m}}{\mathrm{MeV}}\right)^{-1} .
$$

$C_{U}$ cannot be larger than a few units to maintain the perturbative regime nevertheless. Therefore $f_{U e}$ is required to be in the range

$$
f_{U e} \in\left[(3-12) 10^{-8}\left(\frac{m_{U}}{\mathrm{MeV}}\right)^{2}\left(\frac{m_{d m}}{\mathrm{MeV}}\right)^{-1}, 710^{-5}\left(\frac{m_{U}}{\mathrm{MeV}}\right)\left(\frac{\delta a_{e}}{10^{-11}}\right)^{1 / 2}\right] .
$$

Having large values of $C_{U}$ and very small values of $f_{U e}$ however appears quite unnatural as one would naively expect these two couplings to be the same. In any case, however, the new gauge boson appears to be extremely weakly coupled to SM particles. One can now estimate the DM pair production (i.e. $e^{+} e^{-} \rightarrow \mathrm{dm} \mathrm{dm}$ ). We find a cross section that is given by

$$
\sigma_{\mathrm{dmdm}} \propto f_{U l}^{2} C_{U}^{2} / E^{2} \lesssim 610^{-42}\left(\frac{m_{U}}{\mathrm{MeV}}\right)^{4}\left(\frac{E}{\mathrm{GeV}}\right)^{-2} \mathrm{~cm}^{2}
$$

which has to be compared to the $e^{+} e^{-} \rightarrow \nu \nu$, say:

$$
\sigma_{e^{-} e^{+}} \propto G_{F}^{2} E^{2} /(12 \pi) \sim 10^{-39}(E / \mathrm{GeV})^{2} \mathrm{~cm}^{2},
$$

for $E<m_{Z}$.

The $e^{+} e^{-} \rightarrow \mathrm{dm} \mathrm{dm}$ cross section therefore appears very suppressed compared to $e^{+} e^{-} \rightarrow \nu \nu$ at high energy. This explains why it is very difficult to detect it with LEP or TEVATRON experiments. Of course, Light DM would have no direct couplings to the $Z$. Also, due to the structure of the extra $U(1)$, there is no direct modifications of the $Z, W$ boson properties but one has to compute the parameters $S, T, \rho$ of the Standard Model nevertheless.

At much lower energy, on the other hand, the DM pair production cross section becomes quite significative but the associated single photon production (which is a way to 
detect the DM particles) remains still very small compare to other photon production mechanisms (such as the pair electron-positron annihilations into two photons).

The $U$ boson exchange is expected to contribute significantly, on the other hand, to the

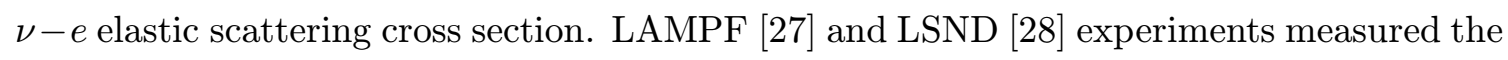
$\nu-e$ cross section with great accuracy. No significative deviation was found so the coupling of the gauge boson with neutrinos (and potentially electrons) should be significantly smaller than the coupling of the gauge boson with DM.

Many other tests remain to be done but Light DM seems to satisfy the most important/obvious ones. There is no theory that predicts such particles though. We shall now present an interesting feature that may seem in favor of a Light Dark Matter candidate.

\section{A bonus for Light Dark Matter}

Recently a bright $511 \mathrm{KeV}$ emission ( $\gamma$-ray) line was observed from the center of our galaxy by the SPI spectrometer on the INTEGRAL (INTErnational Gamma-Ray Astrophysics

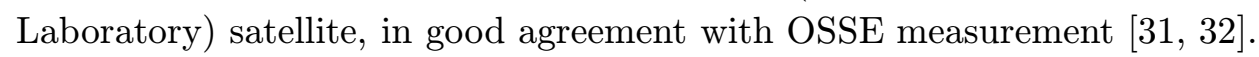

This detection fits reasonably well to a gaussian with full-width half-maximum of $\sim 9^{\circ}$, with a $2 \sigma$ confidence interval of $6^{\circ}-18^{\circ}$.

Positrons in the galactic bulge can be emitted by such

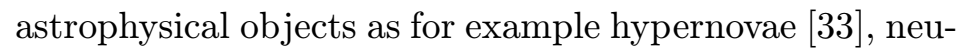
tron stars or black holes [3] 34 in] etc. However, whether these sources can be at the origin of this $511 \mathrm{keV}$ line is still

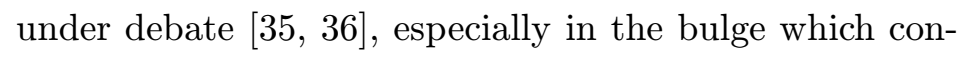
sists exclusively of old, low mass stars. As an alternative explanation, annihilations of light Dark Matter particles

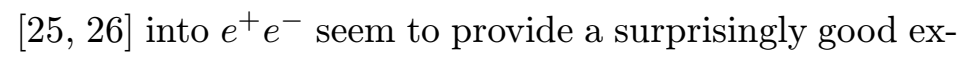
planation for the Integral measurement, if the dark matter halo profile is approximated in the centre of the galaxy as $\rho(r) \propto r^{-\gamma}$ with $\gamma \in[0.4-0.8]$ [30īi $]$. Such a value corresponds to a mild cusp intermediate between the NFW distribution [ $[\bar{\beta} \overline{7}]$ and the profile extrapolated from observations of dwarf and low surface brightness galaxies [30 $\left.{ }^{3} \overline{8}\right]$. This empirically-derived profile is consistent with a density profile that incorporates information on the dark matter contribution constrained by gravitational microlensing

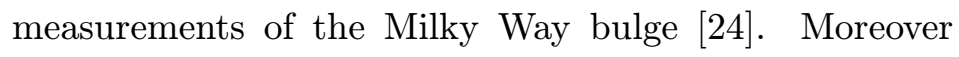
the inferred annihilation cross section is concordant with that inferred from the relic density, without any need to

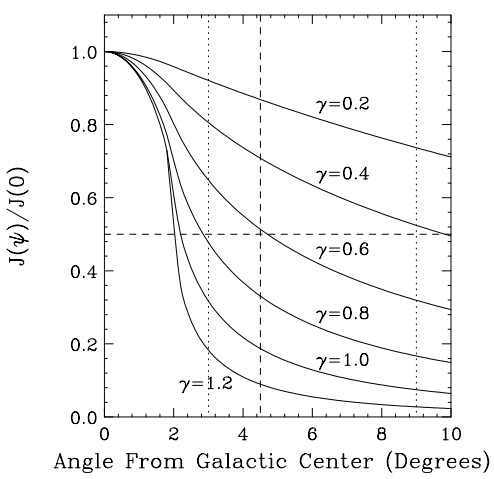

Figure 3: The angular distribution of $\gamma$-rays from DM annihilation averaged over the $2^{\circ}$ angular resolution of SPI for several halo profiles. Shown as vertical dashed and dotted lines are the central value and $2 \sigma$ limits of the angular widths found by SPI. A halo model with $\gamma \sim 0.4-0.8$ is favored. $\backslash[\overline{1} \overline{1} \overline{0}] \bar{c}]$. assume a boost factor, due for example to dark matter clumpiness.

As a result, the flux and angular distribution of the $511 \mathrm{keV}$ line emission seems to be surprisingly well-reproduced by Light DM particles annihilating into $e^{+} e^{-}$, as shown in

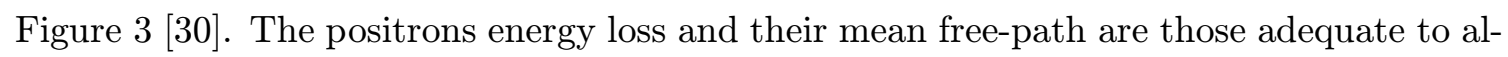
low them to stay in the bulge, allowing for thermalization. In order to discriminate among 
astrophysical sources and Light DM, we propose to to seek a similar signature from low sur-

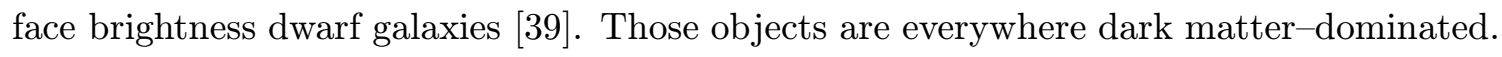
Therefore, if the emission line detected in our galaxy is due to DM annihilations, then one should also detect a $511 \mathrm{keV}$ line from nearby dwarf spheroidals. The flux from the Sagittarius Dwarf Galaxy, in particular, appears to fall within the expected sensitivity of Integral/SPI. It is therefore quite likely that, in the near future, Integral/SPI will be able to rule out or confirm the existence of Light Dark Matter particles.

\section{Conclusions}

There are no real evidences in favor of neutralinos. However, the positrons and gamma ray excess and the possible detection of very High energy gamma rays may appear in favor of very heavy DM particles.

On the other hand, Light Dark Matter seems possible and even quite interesting because of the detection of a $511 \mathrm{keV}$ emission line from the galactic center that can be quite naturally and very simply fitted in a Light DM scenario. A possible smoking gun for light DM could be the detection of a $511 \mathrm{keV}$ emission line from Sagittarius Dwarf Galaxy.

At last, unlike neutralinos which are theoretically motivated, Light DM particles are not. However, if more evidences were validating this scenario, these particles would become very challenging for particle theories.

\section{Acknowlegments}

The author would like to thank the organizers for the opportunity to speak in this conference.

\section{References}

[1] P. Fayet, Phys. Lett. B69, 489 (1977); G. Farrar and P. Fayet, Phys. Lett. B76, 575 (1978).

[2] see for instance D. N. Spergel et al., astro-ph/0302209; A.H. Jaffe et al., Phys. Rev. Lett. 86, 3475 (2001).

[3] B. W. Lee and S. Weinberg, Phys. Rev. Lett. 39, 165 (1977);

[4] H. Goldberg, Phys. Rev. Lett. 50, 1419 (1983).

[5] J. R. Ellis, J. S. Hagelin, D. V. Nanopoulos, K. A. Olive and M. Srednicki, Nucl. Phys. B 238, 453 (1984);

[6] K. Griest, Phys. Rev. Lett. 61, 666 (1988); K. Griest, Phys. Rev. D38, 2357 (1988).

[7] M. Srednicki, R. Watkins and K.A. Olive, Nucl. Phys. B310 (1988) 693.

[8] M. Drees and M. Nojiri, Phys. Rev. D47 (1993) 376.

[9] J. E. Gunn et al., Ap. J. 223, 1015G (1978); M. Davis, M. Lecar, C. Prior, E. Witten, Ap. J. 250, 423 (1981); P. J. E. Peebles, Ap. J. 263L, 1P (1982).

[10] J. Silk, Ap. J. 151, 459 (1968). 
[11] V. K. Narayanan et al., Ap. J. Lett. 543, 103N (2000).

[12] C. L. Bennett et al., "First Year Wilkinson Microwave Anisotropy Probe (WMAP)", astro-ph/0302207.

[13] N. Yoshida et al., astro-ph/0303622.

[14] A. Knebe et al., astro-ph/0302443.

[15] C. Boehm, P. Fayet, R. Schaeffer, Phys. Lett. B518, 8 (2001); Proc. "DARK 2002, 4th Int. Conf. on Dark Matter in Astro and Particle Physics", Cape Town (Springer, 2002), p. 333, astro-ph/0205406; and paper in preparation.

[16] C. Boehm, A. Riazuelo, S. H. Hansen, R. Schaeffer, Phys. Rev. D66, 083505 (2002).

[17] X. Chen, S. Hannestad, R. J. Scherrer, Phys.Rev. D65 (2002) 123515.

[18] C. Boehm, H. Mathis, J. Devriendt, J. Silk, astro-ph/0309652.

[19] A. Bottino, F. Donato, N. Fornengo, S. Scopel, hep-ph/0307303.

[20] D. Hooper, T. Plehn, Phys.Lett. B562 (2003) 18-27.

[21] R. Bernabei et al, astro-ph/0311046.

[22] W. de Boer, M. Herold, C. Sander, V. Zhukov, hep-ph/0312037.

[23] D. Hooper, J. Taylor, J. Silk, hep-ph/0312076.

[24] J.J. Binney, N.W. Evans, astro-ph/0108505.

[25] C. Boehm, T. A. Ensslin and J. Silk, astro-ph/0208458.

[26] C. Boehm and P. Fayet, hep-ph/0305261.

[27] R. C. Allen et al., Phys. Rev. D47, 11 (1993).

[28] LSND coll., Phys. Rev. D63, 112001 (2001).

[29] J. Knodlseder, et.al., Accepted for publication in A\&A, astro-ph/0309442; P. Jean et al., "Early SPI/INTEGRAL measurements of galactic $511 \mathrm{keV}$ line emission astro-ph/0309484.

[30] C. Boehm et al., astro-ph/0309686.

[31] D. D. Dixon et al., astro-ph/9703042; P. A. Milne, J. D. Kurfess, R. L. Kinzer and M. D. Leising, astro-ph/0106157.

[32] D. D. Dixon et al., astro-ph/9703042;

[33] S. E. Woosley and A. Heger, astro-ph/0309165.

[34] R. E. Lingenfelter and R. Ramaty, Positron-Electron Pairs in Astrophysics, eds. M. L. Burns, A. K. Harding and R. Ramaty, AIP Conference Proceedings, 267.

[35] P. A. Milne, L. S. The and M. D. Leising, astro-ph/0104185. P. A. Milne, J. D. Kurfess, R. L. Kinzer and M. D. Leising, New Astron. Rev. 46, 553 (2002) and references therein.

[36] M. Pohl, astro-ph/9807268.

[37] J. F. Navarro, C. S. Frenk and S. D. White, Astrophys. J. 490, 493 (1997).

[38] A. V. Kravtsov, A. A. Klypin, J. S. Bullock and J. R. Primack, astro-ph/9708176.

[39] D. Hooper et al, astro-ph/0311150. 